\title{
Local exact exchange potentials within the all-electron FLAPW method and a comparison with pseudopotential results
}

\author{
Markus Betzinger, ${ }^{1, *}$ Christoph Friedrich, ${ }^{1}$ Stefan Blügel,${ }^{1}$ and Andreas Görling ${ }^{2}$ \\ ${ }^{1}$ Institut für Festkörperforschung and Institute for Advanced Simulation, Forschungszentrum Jülich and JARA, D-52425 Jülich, Germany \\ ${ }^{2}$ Lehrstuhl für Theoretische Chemie, Universität Erlangen-Nürnberg, Egerlandstr. 3, D-91058 Erlangen, Germany
}

(Received 28 September 2010; published 25 January 2011)

\begin{abstract}
We present a general numerical approach to construct local Kohn-Sham potentials from orbital-dependent functionals within the all-electron full-potential linearized augmented-plane-wave (FLAPW) method, in which core and valence electrons are treated on an equal footing. As a practical example, we present a treatment of the orbital-dependent exact-exchange (EXX) energy and potential. A formulation in terms of a mixed product basis, which is constructed from products of LAPW basis functions, enables a solution of the optimizedeffective-potential (OEP) equation with standard numerical algebraic tools and without shape approximations for the resulting potential. We find that the mixed product and LAPW basis sets must be properly balanced to obtain smooth and converged EXX potentials without spurious oscillations. The construction and convergence of the exchange potential are analyzed in detail for diamond. Our all-electron results for $\mathrm{C}, \mathrm{Si}, \mathrm{SiC}, \mathrm{Ge}$, and GaAs semiconductors as well as $\mathrm{Ne}$ and Ar noble-gas solids are in very favorable agreement with plane-wave pseudopotential calculations. This confirms the adequacy of the pseudopotential approximation in the context of the EXX-OEP formalism and clarifies a previous contradiction between FLAPW and pseudopotential results.
\end{abstract}

DOI: 10.1103/PhysRevB.83.045105

\section{INTRODUCTION}

Its wide applicability, accuracy, and computational efficiency have made density-functional theory $(\mathrm{DFT})^{1,2}$ the standard method for describing the ground state of manyelectron systems. The vast majority of practical calculations employ the Kohn-Sham (KS) formalism, ${ }^{3}$ where the interacting many-electron system is mapped onto an auxiliary noninteracting system. In this KS system the noninteracting electrons move in a local effective potential that is defined in such a way that the electron densities of the real and auxiliary systems coincide. This potential is the sum of the external, Hartree, and exchange-correlation (xc) potentials. The latter two contributions take into account the electron-electron interactions including, in an indirect way, all many-body effects. The form of the density-functional for the xc potential, which can further be divided into an exchange and a correlation term, is unknown and must be approximated in practice.

Fortunately, already simple approximations, like the localdensity approximation (LDA) ${ }^{4,5}$ and generalized gradient approximation (GGA), ${ }^{6,7}$ give reliable results for a wide range of materials and properties. Nevertheless, the LDA and GGA suffer from several shortcomings. First, the electrostatic interaction of the electron with the total electron charge, described by the Hartree potential, contains an unphysical interaction of the electron with itself, commonly referred to as Coulomb self-interaction. This extra term should be compensated exactly by an identical term with opposite sign in the exchange potential, in the same manner as in Hartree-Fock theory. However, as the LDA and GGA exchange potentials are only approximate, this cancellation is incomplete and part of the self-interaction remains. This error leads, in particular, to an improper description of localized states, which appear too high in energy and tend to delocalize. Second, the LDA and GGA functionals do not give rise to a discontinuity of the xc potential with respect to changes in the particle number. This discontinuity should in general be finite (and positive), as it corresponds to the difference of the real and
PACS number(s): 71.15.Mb, 71.20.-b, 31.15.E-

the KS band gap. ${ }^{8,9}$ The latter is well known to underestimate experimental gaps by typically $50 \%$ or even more. This is often called the band-gap problem of the LDA and GGA. The significance of the discontinuity for a meaningful prediction of the fundamental band gap is discussed in Refs. 10 and 11.

Functionals that depend explicitly on the electron orbitals, and thus only implicitly on the electron density, form a new generation of $\mathrm{xc}$ functionals. ${ }^{12,13}$ Already the simplest variant, the exact-exchange (EXX) functional, ${ }^{14-16}$ which treats electron exchange exactly but neglects correlation altogether, remedies the aforementioned shortcomings of the more conventional local and semilocal functionals: the Coulomb self-interaction term is exactly canceled, and the local EXX potential exhibits a nonzero discontinuity at integral particle numbers because of the orbital dependence.

After applications to atoms ${ }^{17-19}$ the first implementation for periodic systems was published in 1994 by Kotani, ${ }^{20}$ who employed the atomic sphere approximation within the linearized muffin-tin orbital method. In this approximation, only the spherical part of the potential around each atom is taken into account. The KS band gap turned out to be closer to experiment than in the LDA or GGA. ${ }^{20,21}$ This indicates that the xc discontinuity and the effect of neglecting electron correlation for these systems are roughly of the same magnitude but of different sign and thus tend to cancel each other. Later, results even closer to experimental values were obtained from plane-wave calculations ${ }^{22-24}$ employing the pseudopotential (PP) approximation, which allows accurate treatment of the warped shape of the EXX potential except for the regions close to the atomic nuclei, where the potential is smoothed.

However, when the first all-electron (AE), full-potential results were reported, ${ }^{25}$ they deviated substantially from the $\mathrm{PP}$ values and were also in considerably worse agreement with experiment. In Ref. 25, Sharma et al., who implemented the EXX functional within the full-potential linearized augmented-plane-wave (FLAPW) method, argue that the 
success of EXX in the earlier PP calculations is only an artifact of the neglect of the core-valence exchange interaction. They conclude that treatment of core and valence electrons on the same footing is imperative for a proper EXX calculation. This work started a controversy about the adequacy of the PP approximation in the EXX formalism. Recently, Engel ${ }^{26}$ showed that, on the contrary, AE and PP results for lithium and diamond differ only marginally. The AE calculations were performed with a plane-wave basis by pushing the PP and plane-wave cutoffs to the AE limit. Also recently, Makmal et al. reported a similarly good agreement between $\mathrm{AE}$ and $\mathrm{PP}$ calculations for the diatomic molecules $\mathrm{BeO}$ and $\mathrm{CO}$ using real-space grid approaches. ${ }^{27}$

A final comparison between PP results and results obtained from a genuine $\mathrm{AE}$ approach for periodic systems, such as the FLAPW method, is still missing, though. With this work we want to fill this gap. We present an alternative implementation of the EXX approach within the FLAPW method, which uses a numerical approach different from the one reported in Ref. 25. It employs a specifically designed basis, the mixed product basis, in which the optimized-effective-potential (OEP) equation for the EXX potential is solved. The mixed product functions form an all-electron basis for the products of single-particle wave functions occurring in the OEP equation. Within this approach, both spherical and nonspherical as well as warped interstitial contributions to the EXX potential are fully taken into account. For the example of diamond, we discuss in detail the convergence of the local EXX potential and the resulting KS band gaps with respect to the quality of the mixed product and LAPW basis sets. We demonstrate that a smooth potential and a direct KS band gap very close to the result by Engel ${ }^{26}$ are obtained if the two basis sets are properly balanced. A similar behavior has been reported for Gaussian and plane-wave basis sets. ${ }^{28-30}$ To validate our findings, we also report results for $\mathrm{Si}$, $\mathrm{SiC}, \mathrm{Ge}, \mathrm{GaAs}$, and crystalline $\mathrm{Ne}$ and Ar. For all materials, we find a very good agreement between our AE and previously published plane-wave PP values. We conclude that the large discrepancies found in Ref. 25 cannot be attributed to the core-valence interaction.

The paper is organized as follows. Sections II and III give brief introductions to the theory and the FLAPW method. Our implementation of the EXX functional within the FLAPW program FLEUR ${ }^{31}$ is described in Sec. IV. Section V discusses the convergence of the effective potential for the example of diamond and compares AE EXX KS eigenvalue differences commonly interpreted as transition energies for $\mathrm{C}, \mathrm{Si}, \mathrm{SiC}, \mathrm{Ge}$, $\mathrm{GaAs}$, and crystalline $\mathrm{Ne}$ and Ar with theoretical plane-wave and experimental values from the literature. Finally, we draw our conclusions in Sec. VI.

\section{THEORY}

The KS formalism ${ }^{3}$ of $\mathrm{DFT}^{1}$ relies on an auxiliary system of noninteracting electrons, which move in the spin-dependent local effective potential

$$
V_{\text {eff }}^{\sigma}(\mathbf{r})=V_{\text {ext }}(\mathbf{r})+V_{\mathrm{H}}(\mathbf{r})+V_{\mathrm{xc}}^{\sigma}(\mathbf{r})
$$

with the external, Hartree, and xc potential, respectively. The latter is defined in such a way that the electron spin densities coincide with those of the real interacting system. It is given by the functional derivative of the xc energy functional $E_{\mathrm{xc}}\left[n^{\uparrow}, n^{\downarrow}\right]$ with respect to the electron spin density $n^{\sigma}(\mathbf{r})$ $(\sigma=\uparrow, \downarrow)$,

$$
V_{\mathrm{xc}}^{\sigma}(\mathbf{r})=\frac{\delta E_{\mathrm{xc}}}{\delta n^{\sigma}(\mathbf{r})} .
$$

The orbitals describing the electrons in the auxiliary system obey the KS equations

$$
\left[-\frac{1}{2} \nabla^{2}+V_{\mathrm{eff}}^{\sigma}(\mathbf{r})\right] \varphi_{n \mathbf{k}}^{\sigma}(\mathbf{r})=\epsilon_{n \mathbf{k}}^{\sigma} \varphi_{n \mathbf{k}}^{\sigma}(\mathbf{r}),
$$

where $\varphi_{n \mathbf{k}}^{\sigma}$ denotes the KS orbital of spin $\sigma$, band index $n$, and Bloch vector $\mathbf{k}$. Hartree atomic units are used except where noted otherwise. The electron spin density is given by a sum over the occupied states:

$$
n^{\sigma}(\mathbf{r})=\sum_{\mathbf{k}} \sum_{n}^{\text {occ. }}\left|\varphi_{n \mathbf{k}}^{\sigma}(\mathbf{r})\right|^{2} .
$$

By a summation over Bloch vectors we mean an integration over the Brillouin zone, which is sampled by a finite set of mesh points.

For the conventional LDA and GGA functionals, the xc energy functional depends locally on the spin densities and, in the case of the GGA, on their gradients, and the functional derivative in Eq. (2) translates to a derivative of a function and is evaluated in a straightforward way. However, for orbital-dependent functionals, $E_{\mathrm{xc}}$ depends only indirectly on the electron spin densities: the KS orbitals, which define $E_{\mathrm{xc}}\left[\varphi^{\uparrow}, \varphi^{\downarrow}\right]$, are functionals of the effective potential $V_{\mathrm{eff}}^{\sigma}(\mathbf{r})$ through Eq. (3), and $V_{\text {eff }}^{\sigma}(\mathbf{r})$ is a functional of $n^{\sigma}$. Therefore, one must apply the chain rule to calculate the functional derivative in Eq. (2):

$$
\begin{aligned}
V_{\mathrm{xc}}^{\sigma}(\mathbf{r})= & \sum_{n, \mathbf{k}} \iint\left[\frac{\delta E_{\mathrm{xc}}}{\delta \varphi_{n \mathbf{k}}^{\sigma}\left(\mathbf{r}^{\prime}\right)} \frac{\delta \varphi_{n \mathbf{k}}^{\sigma}\left(\mathbf{r}^{\prime}\right)}{\delta V_{\mathrm{eff}}^{\sigma}\left(\mathbf{r}^{\prime \prime}\right)}+\text { c.c. }\right] \\
& \times \frac{\delta V_{\mathrm{eff}}^{\sigma}\left(\mathbf{r}^{\prime \prime}\right)}{\delta n^{\sigma}(\mathbf{r})} d^{3} r^{\prime} d^{3} r^{\prime \prime},
\end{aligned}
$$

where the sum runs over all KS states present in $E_{\mathrm{xc}}$. Then multiplication with the single-particle spin-density response function

$$
\chi_{\mathrm{s}}^{\sigma}\left(\mathbf{r}, \mathbf{r}^{\prime}\right)=\frac{\delta n^{\sigma}(\mathbf{r})}{\delta V_{\mathrm{eff}}^{\sigma}\left(\mathbf{r}^{\prime}\right)},
$$

integration, and use of $\chi_{\mathrm{s}}^{\sigma}\left(\mathbf{r}, \mathbf{r}^{\prime}\right)=\chi_{\mathrm{s}}^{\sigma}\left(\mathbf{r}^{\prime}, \mathbf{r}\right)$ yield an integral equation for the $\mathrm{xc}$ potential

$$
\begin{aligned}
& \int \chi_{\mathrm{s}}^{\sigma}\left(\mathbf{r}, \mathbf{r}^{\prime}\right) V_{\mathrm{xc}}^{\sigma}\left(\mathbf{r}^{\prime}\right) d^{3} r^{\prime} \\
& =\sum_{\mathbf{k}} \sum_{n} \int\left[\frac{\delta E_{\mathrm{xc}}}{\delta \varphi_{n \mathbf{k}}^{\sigma}\left(\mathbf{r}^{\prime}\right)} \frac{\delta \varphi_{n \mathbf{k}}^{\sigma}\left(\mathbf{r}^{\prime}\right)}{\delta V_{\mathrm{eff}}^{\sigma}(\mathbf{r})}+\text { c.c. }\right] d^{3} r^{\prime} .
\end{aligned}
$$

In this work we employ, as a practical example, the orbitaldependent EXX functional

$$
\begin{aligned}
E_{\mathrm{x}}= & -\frac{1}{2} \sum_{\sigma} \sum_{\mathbf{k}, \mathbf{q}} \sum_{n, n^{\prime}}^{\mathrm{occ}} \iint d^{3} r d^{3} r^{\prime} \\
& \times \frac{\varphi_{n \mathbf{k}}^{\sigma *}(\mathbf{r}) \varphi_{n^{\prime} \mathbf{q}}^{\sigma}(\mathbf{r}) \varphi_{n^{\prime} \mathbf{q}}^{\sigma *}\left(\mathbf{r}^{\prime}\right) \varphi_{n \mathbf{k}}^{\sigma}\left(\mathbf{r}^{\prime}\right)}{\left|\mathbf{r}-\mathbf{r}^{\prime}\right|},
\end{aligned}
$$


whose functional derivative with respect to the $\mathrm{KS}$ wave functions is given by the well-known Hartree-Fock expression

$$
\frac{\delta E_{\mathrm{x}}}{\delta \varphi_{n \mathbf{k}}^{\sigma}\left(\mathbf{r}^{\prime}\right)}=\int \varphi_{n \mathbf{k}}^{\sigma *}\left(\mathbf{r}^{\prime \prime}\right) V_{\mathrm{x}, \mathrm{NL}}^{\sigma}\left(\mathbf{r}^{\prime \prime}, \mathbf{r}^{\prime}\right) d^{3} r^{\prime \prime}
$$

with

$$
V_{\mathrm{x}, \mathrm{NL}}^{\sigma}\left(\mathbf{r}^{\prime \prime}, \mathbf{r}^{\prime}\right)=-\sum_{\mathbf{q}} \sum_{n^{\prime}}^{\text {occ. }} \frac{\varphi_{n^{\prime} \mathbf{q}}^{\sigma}\left(\mathbf{r}^{\prime \prime}\right) \varphi_{n^{\prime}}^{\sigma *}\left(\mathbf{r}^{\prime}\right)}{\left|\mathbf{r}^{\prime}-\mathbf{r}^{\prime \prime}\right|}
$$

First-order perturbation theory yields the wave-function response

$$
\frac{\delta \varphi_{n \mathbf{k}}^{\sigma}(\mathbf{r})}{\delta V_{\mathrm{eff}}^{\sigma}\left(\mathbf{r}^{\prime}\right)}=\sum_{n^{\prime}(\neq n)} \frac{\varphi_{n^{\prime} \mathbf{k}}^{\sigma *}\left(\mathbf{r}^{\prime}\right) \varphi_{n \mathbf{k}}^{\sigma}\left(\mathbf{r}^{\prime}\right)}{\epsilon_{n \mathbf{k}}^{\sigma}-\epsilon_{n^{\prime} \mathbf{k}}^{\sigma}} \varphi_{n^{\prime} \mathbf{k}}^{\sigma}(\mathbf{r})
$$

and, together with Eq. (4), the spin-density response function

$$
\chi_{s}^{\sigma}\left(\mathbf{r}, \mathbf{r}^{\prime}\right)=2 \sum_{\mathbf{k}} \sum_{n}^{\text {occ. unocc. }} \sum_{n^{\prime}} \frac{\varphi_{n \mathbf{k}}^{\sigma *}(\mathbf{r}) \varphi_{n^{\prime} \mathbf{k}}^{\sigma}(\mathbf{r}) \varphi_{n^{\prime} \mathbf{k}}^{\sigma *}\left(\mathbf{r}^{\prime}\right) \varphi_{n \mathbf{k}}^{\sigma}\left(\mathbf{r}^{\prime}\right)}{\epsilon_{n \mathbf{k}}^{\sigma}-\epsilon_{n^{\prime} \mathbf{k}}^{\sigma}},
$$

where time-reversal symmetry has been used. Using Eqs. (9), (11), and (12) the integral equation [Eq. (7)] turns into

$$
\int \chi_{\mathrm{s}}^{\sigma}\left(\mathbf{r}, \mathbf{r}^{\prime}\right) V_{\mathrm{x}}^{\sigma}\left(\mathbf{r}^{\prime}\right) d^{3} r^{\prime}=t^{\sigma}(\mathbf{r})
$$

with

$$
\begin{aligned}
t^{\sigma}(\mathbf{r}) & =\frac{\delta E_{\mathrm{x}}}{\delta V_{\mathrm{eff}}^{\sigma}(\mathbf{r})} \\
& =2 \sum_{\mathbf{k}} \sum_{n}^{\text {occ. unocc. }} \sum_{n^{\prime}}\left[\left\langle\varphi_{n \mathbf{k}}^{\sigma}\left|V_{\mathrm{x}, \mathrm{NL}}^{\sigma}\right| \varphi_{n^{\prime} \mathbf{k}}^{\sigma}\right\rangle \frac{\varphi_{n^{\prime} \mathbf{k}}^{\sigma *}(\mathbf{r}) \varphi_{n \mathbf{k}}^{\sigma}(\mathbf{r})}{\epsilon_{n \mathbf{k}}^{\sigma}-\epsilon_{n^{\prime} \mathbf{k}}^{\sigma}}\right]
\end{aligned}
$$

and

$$
\left\langle\varphi_{n \mathbf{k}}^{\sigma}\left|V_{\mathrm{x}, \mathrm{NL}}^{\sigma}\right| \varphi_{n^{\prime} \mathbf{k}}^{\sigma}\right\rangle=\iint \varphi_{n \mathbf{k}}^{\sigma *}(\mathbf{r}) V_{\mathrm{x}, \mathrm{NL}}^{\sigma}\left(\mathbf{r}, \mathbf{r}^{\prime}\right) \varphi_{n^{\prime} \mathbf{k}}^{\sigma}\left(\mathbf{r}^{\prime}\right) d^{3} r d^{3} r^{\prime} .
$$

In this form the integral equation is called the OEP equation and goes back to Sharp and Horton, ${ }^{32}$ who derived Eq. (13) as a result of a variational minimization of the Hartree-Fock total energy under the additional constraint that the orbitals experience a local rather than a nonlocal potential. Sahni et $a .^{33}$ finally realized that the OEP approach is equivalent to the construction of the local EXX potential within the KS formalism.

\section{FLAPW METHOD}

The LAPW basis ${ }^{34-36}$ is constructed from piecewise defined functions to deal, at the same time, with the atomic-like potential close to the nuclei and the smooth potential in the region far away from the nuclei. For this purpose, space is partitioned into nonoverlapping atom-centered muffin-tin (MT) spheres and the remaining interstitial region (IR), where the smoothness of the potential allows to employ plane waves as basis functions. At the MT sphere boundaries, these plane waves are matched in value and first radial derivative to linear combinations of spin-dependent MT solutions $u_{l 0}^{a \sigma}(r) Y_{l m}(\hat{\mathbf{r}})$ of the radial scalar-relativistic Dirac equation and their energy derivatives $u_{l 1}^{a \sigma}(r) Y_{l m}(\hat{\mathbf{r}})$ using the spherical average of the effective potential and predefined energy parameters that lie in the energy range of the occupied states. Here, $Y_{l m}(\hat{\mathbf{r}})$ denote the spherical harmonics, $\mathbf{r}$ is measured from the MT center of atom $a$, and $\hat{\mathbf{r}}=\mathbf{r} / r$ is a unit vector. This gives the LAPW basis functions

$$
\phi_{\mathbf{k G}}^{\sigma}(\mathbf{r})=\left\{\begin{array}{cc}
\frac{1}{\sqrt{\Omega}} \exp [i(\mathbf{k}+\mathbf{G}) \cdot \mathbf{r}] & \text { if } \quad \mathbf{r} \in \operatorname{IR} \\
\sum_{l=0}^{l_{\max }} \sum_{m=-l}^{l} \sum_{p=0}^{1} A_{l m p}^{\sigma}(\mathbf{k}, \mathbf{G}) u_{l p}^{a \sigma}\left(\left|\mathbf{r}-\mathbf{R}_{a}\right|\right) Y_{l m}\left(\mathbf{r} \widehat{-\mathbf{R}_{a}}\right) & \text { if } \quad \mathbf{r} \in \operatorname{MT}(a)
\end{array}\right.
$$

for the valence electrons with the unit-cell volume $\Omega$ and reciprocal lattice vectors G. For a practical calculation, cutoff values for the reciprocal lattice vectors $|\mathbf{k}+\mathbf{G}| \leqslant$ $G_{\max }$ and the angular momentum $l \leqslant l_{\max }$ are employed. The core states are obtained by solving the fully relativistic Dirac equation with the spherical average of the effective potential.

The basis functions, defined in Eq. (16), can represent accurately only those wave functions whose energies are sufficiently close to the energy parameters, which are usually located in the valence-band region. For a precise description of semicore and high-lying unoccupied states that are far away from the energy parameters, the basis must be augmented and local orbitals ${ }^{37-39}$ are currently the best-developed technique. Let us assume that we want to improve the basis for states with an angular momentum $l$ around an energy $\epsilon^{\text {lo }}$. Then we construct an additional radial function $u_{l}^{a \sigma}\left(r, \epsilon^{\mathrm{lo}}\right)$ from the radial scalar-relativistic Dirac equation with the energy parameter $\epsilon^{\text {lo }}$ and form a linear combination $u_{l p}^{a \sigma}(r) Y_{l m}(\hat{\mathbf{r}})(p \geqslant 2$; the index $p$ is a label numbering the basis functions for a given $l, a$, and $\sigma)$ from $u_{l}^{a \sigma}\left(r, \epsilon^{\mathrm{lo}}\right)$ and the radial functions $u_{l 0}^{a \sigma}(r)$ and $u_{l 1}^{a \sigma}(r)$, already defined, such that $u_{l p}^{a \sigma}(r)$ is normalized and its value and radial derivative vanish at the MT boundary. In this way, the local orbital $u_{l p}^{a \sigma}(r) Y_{l m}(\hat{\mathbf{r}})$ is completely confined to the MT sphere and need not be matched to a plane wave outside. For semicore states, which are nearly dispersionless, the energy parameter $\epsilon^{\text {lo }}$ is fixed at the semicore energy level. For the unoccupied states we use energy parameters chosen such that the solutions of the radial scalar-relativistic Dirac equation fulfill

$$
\left.\frac{d}{d r} \ln \left[u_{l}^{a \sigma}\left(r, \epsilon^{\mathrm{lo}}\right)\right]\right|_{r=S}=-(l+1)
$$

at the MT sphere boundary $r=S$, following a procedure proposed in Ref. 40. This condition yields, for each $l$ quantum number, a series of orthogonal solutions of increasing energies. 
We use the resulting local orbitals to converge the LAPW basis in a systematic way.

\section{IMPLEMENTATION}

To solve the integral equation [Eq. (13)], we introduce a basis $\left\{M_{I}(\mathbf{r})\right\}$ that reformulates the equation as a linear-algebra problem,

$$
\sum_{J} \chi_{\mathrm{s}, I J}^{\sigma} V_{\mathrm{x}, J}^{\sigma}=t_{I}^{\sigma}
$$

which can be solved for the exchange potential $V_{\mathrm{x}}^{\sigma}$ by matrix inversion of $\chi_{\mathrm{s}}^{\sigma}$ applying standard numerical techniques. As all quantities appearing in Eq. (13) are defined in terms of wavefunction products, the basis should be constructed foremost of products of LAPW basis functions. In recent publications we have already used such a mixed product basis (MPB), which was first proposed by Kotani and van Schilfgaarde, ${ }^{41}$ to implement hybrid functionals ${ }^{42}$ and the $G W$ approximation $^{43}$ as well as to calculate EELS spectra. ${ }^{44}$ However, we introduce a slightly modified version for the present purpose: (1) as the potential is strictly periodic, the MPB may be restricted to $\mathbf{k}=\mathbf{0}$; (2) we add the atomic EXX potential as a basis function; and (3) we make the functions $M_{I}(\mathbf{r})$ continuous over the whole space.

The construction of the MPB and the implementation of the spin-density response function $\chi_{s, I J}^{\sigma}$ and $t_{I}^{\sigma}$ are described in Secs. IV A and IV B, respectively. Numerical tests of the implementation are reported in Sec. IV C.

\section{A. Mixed product basis}

The MPB consists of plane waves in the IR and MT functions $M_{L P}^{a}(r) Y_{L M}(\hat{\mathbf{r}})$ in the spheres that derive from products of the functions $u_{l p}^{a \sigma}(r) Y_{l m}(\hat{\mathbf{r}})$. As in the LAPW basis, cutoff values $G_{\max }^{\prime}$ for the interstitial plane waves and $L_{\max }$ for the angular momentum quantum numbers $L$ are employed. For mathematical details of the construction of the MPB, we refer the reader to our previous publications, Refs. 42-44. Here, we place emphasis on the modifications for the present implementation of the EXX-OEP method.

From EXX-OEP calculations of atoms it is known that the local exchange potential shows pronounced humps that reflect the atomic shell structure. ${ }^{18}$ As the electron orbitals contract spatially for atoms with larger atomic numbers, these humps move closer and closer to the atomic nucleus. Near the nucleus, the exchange potential of a periodic crystal resembles that of the corresponding atom, because the long-range exchange interactions with electrons on neighboring atoms contribute only a slowly varying potential there. Therefore, we augment the MPB with the spherical exchange potential from an atomic EXX-OEP calculation performed with the relativistic atomic structure program RELKS. ${ }^{45-47}$ It is added to the set of spherical MT functions $(L=0)$. The rest of the basis must then describe only the difference between the atomic and the crystal exchange potential. In this way all nonlocal exchange contributions are fully taken into account.

To avoid discontinuities of the resulting potential at the MT sphere boundaries, we form linear combinations of the MT functions and interstitial plane waves that are continuous in value and first derivative there. In analogy to the construction of the LAPW basis (Sec. III), two radial functions per $l m$ channel are used to augment the interstitial plane waves in the MT spheres, while the remaining functions are combined to form local orbitals. We note that there are usually far more than two radial functions per $l m$ channel in the MPB. We also note that such a construction was not needed in our earlier implementations.

\section{B. Spin-density response function and $t_{I}^{\sigma}(\mathrm{r})$}

In the MPB the spin-density response function in Eq. (12) and $t_{I}^{\sigma}(\mathbf{r})$ in Eq. (14) become

$$
\chi_{\mathrm{s}, I J}^{\sigma}=2 \sum_{\mathbf{k}} \sum_{n}^{\text {occ. unocc. }} \sum_{n^{\prime}} \frac{\left\langle M_{I} \varphi_{n^{\prime} \mathbf{k}}^{\sigma} \mid \varphi_{n \mathbf{k}}^{\sigma}\right\rangle\left\langle\varphi_{n \mathbf{k}}^{\sigma} \mid \varphi_{n^{\prime} \mathbf{k}}^{\sigma} M_{J}\right\rangle}{\epsilon_{n \mathbf{k}}^{\sigma}-\epsilon_{n^{\prime} \mathbf{k}}^{\sigma}}
$$

and

$$
t_{I}^{\sigma}=2 \sum_{\mathbf{k}} \sum_{n}^{\text {occ. unocc. }} \sum_{n^{\prime}}\left\langle\varphi_{n \mathbf{k}}^{\sigma}\left|V_{\mathrm{x}, \mathrm{NL}}^{\sigma}\right| \varphi_{n^{\prime} \mathbf{k}}^{\sigma}\right\rangle \frac{\left\langle M_{I} \varphi_{n^{\prime} \mathbf{k}}^{\sigma} \mid \varphi_{n \mathbf{k}}^{\sigma}\right\rangle}{\epsilon_{n \mathbf{k}}^{\sigma}-\epsilon_{n^{\prime} \mathbf{k}}^{\sigma}} .
$$

Both core and valence states are taken into account in the sums over the occupied states in Eqs. (19) and (20).

As said before, solving Eq. (18) for the exchange potential involves the matrix inversion of $\chi_{s, I J}^{\sigma}$. The Hohenberg and Kohn theorem guarantees that the response function is invertible except for variations of the potential given by an addition of a constant. The latter restriction gives rise to a constant eigenfunction of $\chi_{s, I J}^{\sigma}$ with eigenvalue 0 , which we eliminate from the outset by orthogonalizing all MPB functions to a constant function such that variations in the potential by a constant are excluded.

Equation (20) contains the matrix elements of the nonlocal exchange potential [Eq. (15)] between occupied (core and valence) and unoccupied states. In a recent publication, we described an efficient scheme to calculate the valence-valence and valence-conduction matrix elements within the FLAPW method. ${ }^{42}$ For the present implementation, this scheme has been extended to the core-conduction matrix elements. Furthermore, spatial and time-reversal symmetries are exploited to restrict the k-point sums to the irreducible wedge of the Brillouin zone in Eqs. (19) and (20). ${ }^{42,43}$

\section{Numerical tests}

In this section, we present numerical tests of the spindensity response function, the function $t_{I}^{\sigma}(\mathbf{r})$, and the resulting exchange potential. According to Eqs. (2), (6), and (14), all three quantities are functional derivatives of the form $\frac{\delta A(\mathbf{r})}{\delta B\left(\mathbf{r}^{\prime}\right)}$. Thus, they describe the linear response of a quantity $A$ with respect to changes of a quantity $B$.

For the case of diamond, we calculate the changes $\Delta n(\mathbf{r})$ and $\Delta E_{\mathrm{x}}$ that result from an explicit perturbation $V_{\mathrm{per}}(\mathbf{r})=\alpha \sum_{I} V_{\mathrm{per}, I} M_{I}(\mathbf{r})$, where $V_{\mathrm{per}, I}$ are random numbers, by exact diagonalization of the perturbed Hamiltonian and check whether they correspond to $\Delta n^{\operatorname{lin}}(\mathbf{r})=\int \chi_{\mathrm{s}}\left(\mathbf{r}, \mathbf{r}^{\prime}\right) V_{\mathrm{per}}\left(\mathbf{r}^{\prime}\right) d^{3} r^{\prime}, \Delta E_{\mathrm{x}}^{\operatorname{lin}}=\int t\left(\mathbf{r}^{\prime}\right) V_{\mathrm{per}}\left(\mathbf{r}^{\prime}\right) d^{3} r^{\prime}$, and $\Delta E_{\mathrm{x}}^{\text {lin }}=\int V_{\mathrm{x}}\left(\mathbf{r}^{\prime}\right) \Delta n(\mathbf{r}) d^{3} r$ up to linear order in $\alpha$. 
TABLE I. Numerical test for the response function $\chi_{\mathrm{s}}\left(\mathbf{r}, \mathbf{r}^{\prime}\right)=$ $\delta n(\mathbf{r}) / \delta V_{\mathrm{s}}\left(\mathbf{r}^{\prime}\right)$ of diamond. The exact response of the density $\Delta n(\mathbf{r})$ is compared with its linear approximation $\Delta n^{\operatorname{lin}}(\mathbf{r})=\sum n_{I}^{\operatorname{lin}} M_{I}(\mathbf{r})$ with $n_{I}^{\text {lin }}=\alpha \sum_{J} \chi_{\mathrm{s}, I J} V_{\mathrm{per}, J}$. The L2 norm $\left[\int\left|\Delta n^{\operatorname{lin}}(\mathbf{r})-\Delta n(\mathbf{r})\right|^{2} d^{3} r\right]^{1 / 2}$ clearly shows a quadratic dependence on the perturbation strength $\alpha$.

\begin{tabular}{cccc}
\hline \hline$\alpha$ & 0.01 & 0.001 & 0.0001 \\
\hline$\left|\Delta n^{\text {lin }}-\Delta n\right|$ & $2.16710 \times 10^{-4}$ & $2.17018 \times 10^{-6}$ & $2.17035 \times 10^{-8}$ \\
\hline \hline
\end{tabular}

Tables I, II, and III show that, indeed, the differences $\left|\Delta n^{\text {lin }}-\Delta n\right|$ and $\left|\Delta E_{\mathrm{x}}^{\text {lin }}-\Delta E_{\mathrm{x}}\right|$ depend quadratically on the perturbation strength $\alpha$ which confirms the validity of our implementation.

\section{RESULTS AND DISCUSSION}

In this section, we present results for a variety of semiconductors and insulators obtained with our implementation of the EXX-OEP approach within the FLAPW method. In particular, we demonstrate for the case of diamond that a smooth and physical EXX potential requires a balance of the basis sets: the LAPW basis for the wave functions must be converged with respect to a given MPB until the EXX potential does not change anymore. This is somewhat counterintuitive and in contrast to our implementation of the hybrid functionals where, conversely, the MPB must be converged for a given LAPW basis. A similar behavior has been found in implementations employing plane-wave and Gaussian basis sets. ${ }^{28,29}$ We analyze and explain this point later in this section.

Figure 1 shows the local EXX potential on lines connecting two neighboring carbon atoms along the [111] [Fig. 1(a)] and the [100] [Fig. 1(b)] directions; see Fig. 2. A $4 \times 4 \times 4$ k-point sampling is employed, and the MPB parameters are $G_{\max }^{\prime}=3.4 a_{0}^{-1}\left(a_{0}\right.$ is the Bohr radius) and $L_{\max }=4$, giving rise to five $s$-, four $p$-, four $d$-, three $f$-, and two $g$-type radial functions per atom. These cutoff values are well below those of the LAPW basis, $l_{\max }=6$ and $G_{\max }=4.2 a_{0}^{-1}$, which reflects the relative smoothness of the potential compared with the shape of the wave functions. However, if we only use the conventional basis of augmented plane waves, defined in Eq. (16), the potential (dashed lines) shows an overpronounced intershell hump and tends to an unphysical positive value close to the atomic nucleus $(r=0)$. This is a case where the basis sets are unbalanced. In particular, the LAPW basis lacks flexibility in the MT spheres, as becomes obvious when we add local

TABLE II. Same as Table I, for $t(\mathbf{r})=\delta E_{\mathrm{x}} / \delta V_{\mathrm{s}}(\mathbf{r})$. The difference of the exact response $\Delta E_{\mathrm{x}}$ of the exchange energy and its linear approximation $\Delta E_{\mathrm{x}}^{\text {lin }}=\alpha \sum_{I} t_{I} V_{\mathrm{per}, I}$ depends quadratically on $\alpha$.

\begin{tabular}{lccc}
\hline \hline$\alpha$ & 0.01 & 0.001 & 0.0001 \\
\hline$\Delta E_{\mathrm{x}}$ & 1.69455 & $1.74639 \times 10^{-1}$ & $1.75171 \times 10^{-2}$ \\
$\Delta E_{\mathrm{x}}^{\operatorname{lin}}$ & 1.75230 & $1.75230 \times 10^{-1}$ & $1.75230 \times 10^{-2}$ \\
$\left|\Delta E_{\mathrm{x}}^{\operatorname{lin}}-\Delta E_{\mathrm{x}}\right|$ & $5.77479 \times 10^{-2}$ & $5.90882 \times 10^{-4}$ & $5.92211 \times 10^{-6}$ \\
\hline \hline
\end{tabular}

TABLE III. Same as Table II, for $V_{\mathrm{x}}(\mathbf{r})=\delta E_{\mathrm{x}} / \delta n(\mathbf{r})$ with $\Delta E_{\mathrm{x}}^{\operatorname{lin}}=\sum_{I} V_{\mathrm{x}, I} \int M_{I}^{*}(\mathbf{r}) \Delta n(\mathbf{r}) d^{3} r$.

\begin{tabular}{lccc}
\hline \hline$\alpha$ & 0.01 & 0.001 & 0.0001 \\
\hline$\Delta E_{\mathrm{x}}$ & 1.69455 & $1.74639 \times 10^{-1}$ & $1.75171 \times 10^{-2}$ \\
$\Delta E_{\mathrm{x}}^{\operatorname{lin}}$ & 1.67924 & $1.74479 \times 10^{-1}$ & $1.75155 \times 10^{-2}$ \\
$\left|\Delta E_{\mathrm{x}}^{\operatorname{lin}}-\Delta E_{\mathrm{x}}\right|$ & $1.53093 \times 10^{-2}$ & $1.59919 \times 10^{-4}$ & $1.60625 \times 10^{-6}$ \\
\hline \hline
\end{tabular}

orbitals, which are nonzero only in the MT spheres. We find that six local orbitals for each $l m$ channel with $l=0, \ldots, 5$ and $|m| \leqslant l$, placed at higher energies according to the prescription described in Sec. III, are needed to converge the local EXX potential. This is reasonable since, with the cutoff $L_{\max }=4$, the occupied $2 s$ and $2 p$ states of diamond couple maximally to the $l=5$ contribution of the unoccupied states. With so many local orbitals, the number of basis functions is increased by
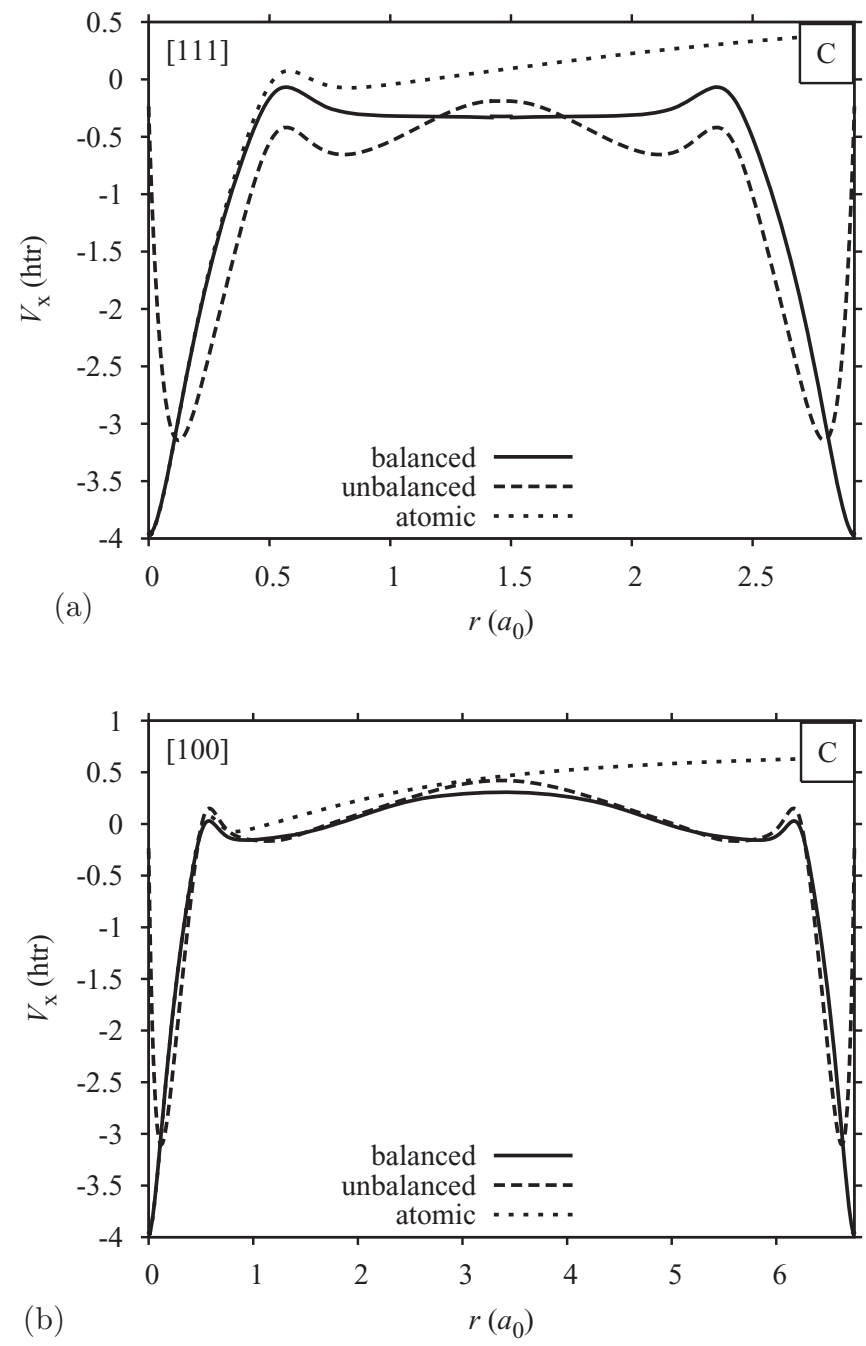

FIG. 1. Local EXX potential of diamond on lines starting and ending at atomic nuclei along (a) the [111] and (b) the [100] directions. Dashed and solid lines correspond to cases where the MPB and LAPW basis sets are unbalanced and balanced, respectively. For comparison, we also show the atomic EXX potential of carbon as dotted lines, shifted to align with the crystal EXX potential at the atomic nucleus at $r=0$. 


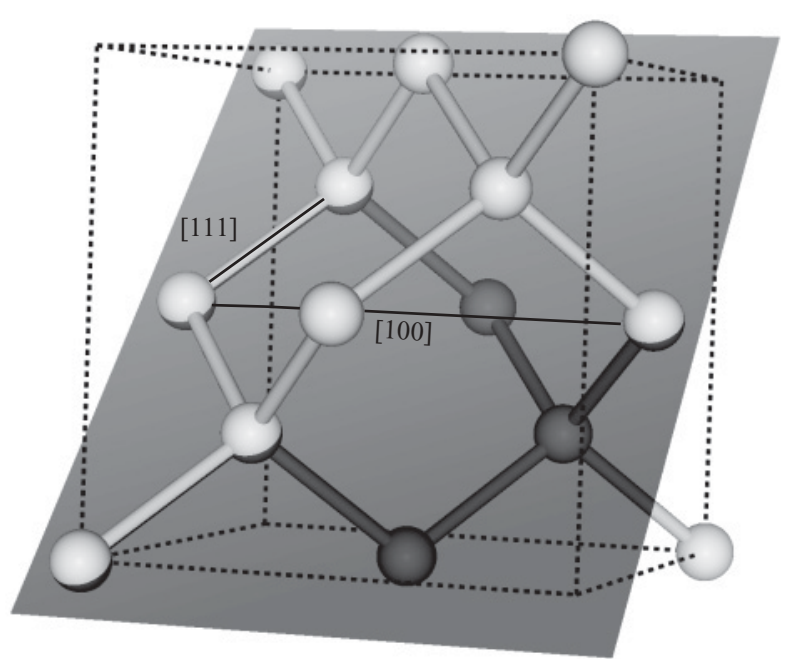

FIG. 2. Cubic unit cell of diamond with the $(01 \overline{1})$ plane. Solid lines indicate the lines connecting two carbon atoms along the [111] and [100] directions.

roughly a factor of 5: there are about 100 augmented plane waves (the exact number depends on the $\mathbf{k}$ point) and 432 additional local orbitals. All resulting KS bands, about 530, are taken into account in the sums of Eqs. (12) and (14). The resulting potential is shown as solid lines in Fig. 1 and looks smooth and physical. It is remarkable that, even for diamond, it takes so much effort to converge the EXX potential since, in conventional LDA or GGA calculations, diamond is treated readily with a very modest LAPW basis, without any local orbitals.

Before analyzing this point in more detail, we want to identify the MPB functions that contribute most to the MT part of the potential. Not surprisingly, the function that corresponds to the atomic EXX potential gets the largest weight. In fact, close to the atomic nuclei this function (dotted lines in Fig. 1) and its bulk counterpart are indistinguishable. They deviate more and more toward the MT sphere boundary $\left(S=1.42 a_{0}\right)$, where the atomic EXX potential already enters the typical $1 / r$ behavior, while the crystal EXX potential is periodic. The second-largest contribution comes from the constant MT function, which helps to align the MT potential to the interstitial one. We note that there is no ambiguity with respect to adding a constant to the potential over the whole space, since the constant function has been eliminated explicitly from the MPB (see Sec. IV B), giving rise to the condition $\int V_{\mathrm{x}}(\mathbf{r}) d^{3} r=0$.

So far, we have only discussed the MT potential, whose proper convergence requires additional local orbitals in the spheres. We find an analogous behavior for the interstitial potential. As shown in Fig. 3, the cutoff radius of the reciprocal lattice vectors included in the LAPW basis set must be converged with respect to that of the MPB. To show this effect clearly, the latter was chosen much larger than necessary, $G_{\max }^{\prime}=5.8 a_{0}^{-1}$. Similarly to the MT potential, the interstitial potential exhibits spurious oscillations in the underconverged cases. Only if the $G_{\max }$ of the LAPW basis is large enough, $G_{\max } \gtrsim 5.0 a_{0}^{-1}$, the oscillations are suppressed and a smooth potential is obtained. Furthermore, Fig. 3 shows that, in

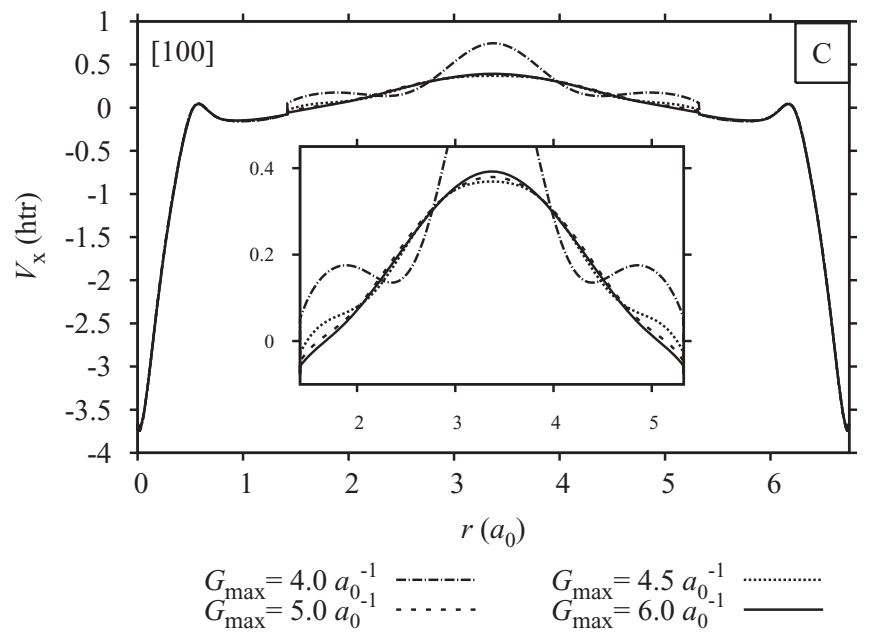

FIG. 3. Convergence of the interstitial EXX potential on a line connecting two carbon atoms along the [100] direction for four reciprocal cutoff radii $G_{\max }$ of the LAPW basis. The corresponding MPB cutoff is fixed at $5.8 a_{0}^{-1}$.

underconverged cases, the potential is not continuous at the MT sphere boundary because the oscillatory potentials possess large-G Fourier coefficients and require spherical harmonics beyond $L_{\max }=4$ in the spheres for a proper matching. Fortunately, the converged EXX potential is a smooth function, and already moderate reciprocal cutoff radii $G_{\max }^{\prime}$ of the MPB are sufficient, typically $75 \%$ of the usual LAPW cutoff. For diamond, for example, the combination of $G_{\max }^{\prime}=3.4 a_{0}^{-1}$ and $G_{\max }=4.2 a_{0}^{-1}$ leads to stable results.

An overall view of the MT and the interstitial exchange potential on the diamond (01) plane is shown in Fig. 4(a) as a contour plot. The plane is displayed in Fig. 2. It contains the connecting lines along [111] and [100] that correspond to Fig. 1. We see that in the regions close to the atomic nuclei, the potential is predominantly spherical. However, toward the MT sphere boundaries the potential becomes strongly anisotropic and matches continuously to the warped interstitial potential, which is far from constant also. In fact, the nonsphericity of the EXX potential is considerably more pronounced than in the LDA potential, Fig. 4(b). The latter is similar in shape to the electron density distribution [cf. Fig. 5(a)], of which it is a direct function $V_{\mathrm{x}}^{\mathrm{LDA}}(\mathbf{r}) \propto n(\mathbf{r})^{1 / 3}$. The EXX potential, in contrast, incorporates the full nonlocality of the EXX functional, which makes it much more corrugated than the LDA one, in particular, in the MT spheres, where the KS orbitals are highly oscillatory. All this stresses the importance of a full-potential treatment within the EXX-OEP approach.

The LDA potential corresponds in each point $\mathbf{r}$ to the exchange potential of the homogeneous electron gas with an electron density that equals the local electron density $n(\mathbf{r})$ of the real system. Thus, by construction it is exact for the homogeneous electron gas but misses the effects of density variations. The EXX potential, in contrast, takes all density variations exactly into account. Thus, the differences between Figs. 4(a) and 4(b) must be attributed to the influence of the density inhomogeneities on the exchange potential. This influence is particularly large in regions where the 


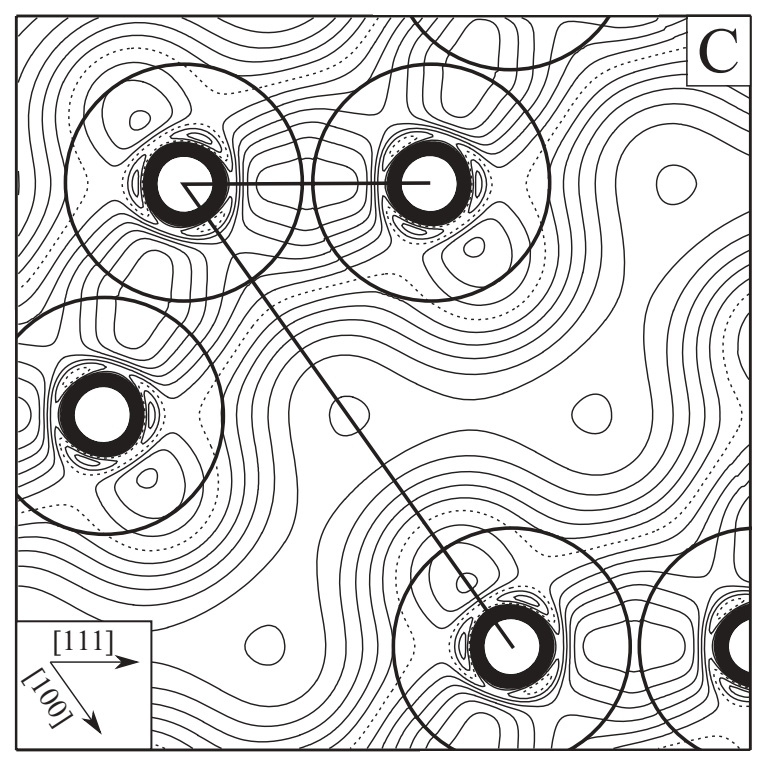

(a)

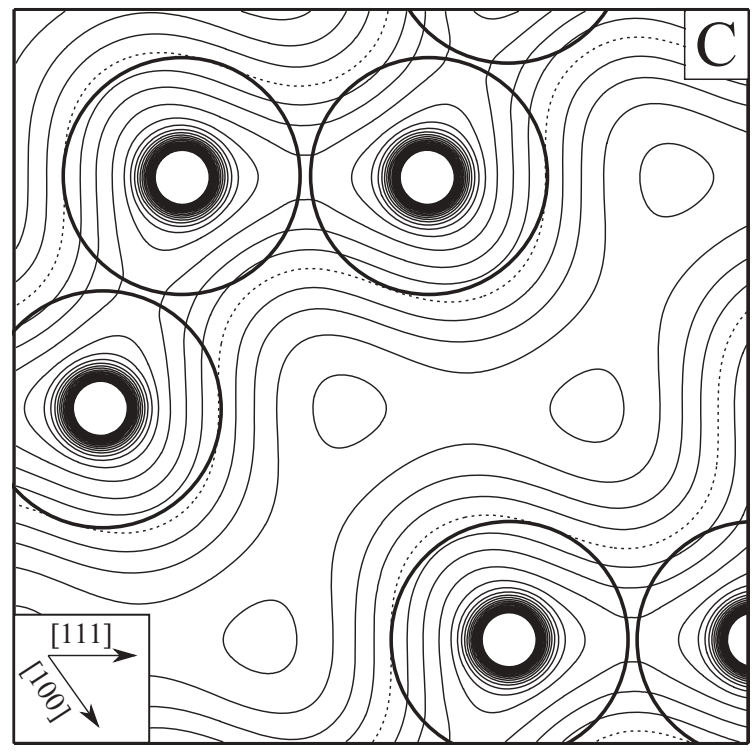

(b)

FIG. 4. Two-dimensional plot of (a) the local EXX and (b) the LDA exchange potential on the $(01 \overline{1})$ plane of diamond. Both potentials are shifted such that $\int V_{\mathrm{x}}(\mathbf{r}) d^{3} r=0$. The contour lines start at $V_{\mathrm{x}}=-1.00 \mathrm{htr}$ and have an interval of $0.05 \mathrm{htr}$. The contour line with the maximal value is found (a) at $V_{\mathrm{x}}=0.30 \mathrm{htr}$ and (b) at $V_{\mathrm{x}}=0.20 \mathrm{htr}$. The dotted line corresponds to $V_{\mathrm{x}}=0$. The MT sphere boundaries and the lines corresponding to Fig. 1 are indicated.

density varies a lot, that is, close to the atomic nuclei, while in the interstitial region the two potentials are more similar.

The differences in the exchange potentials naturally affect the electron density distribution. The EXX electron density, Fig. 5(a), clearly shows a pronounced contraction of the electron distribution compared with the LDA one. This is a direct consequence of the self-interaction error, which is eliminated in the EXX approach, while it gives rise to an unphysical delocalization in the case of the LDA potential.

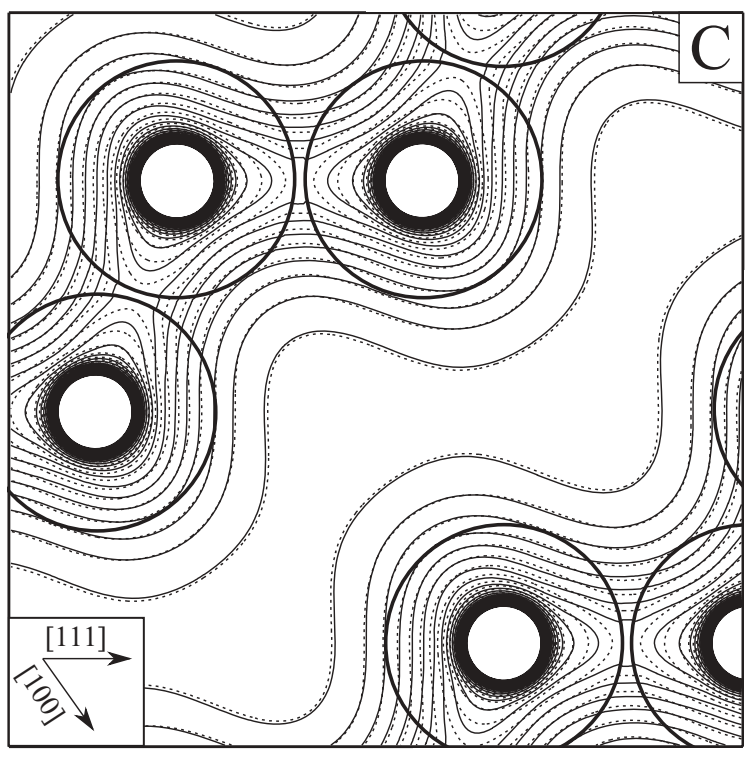

(a)

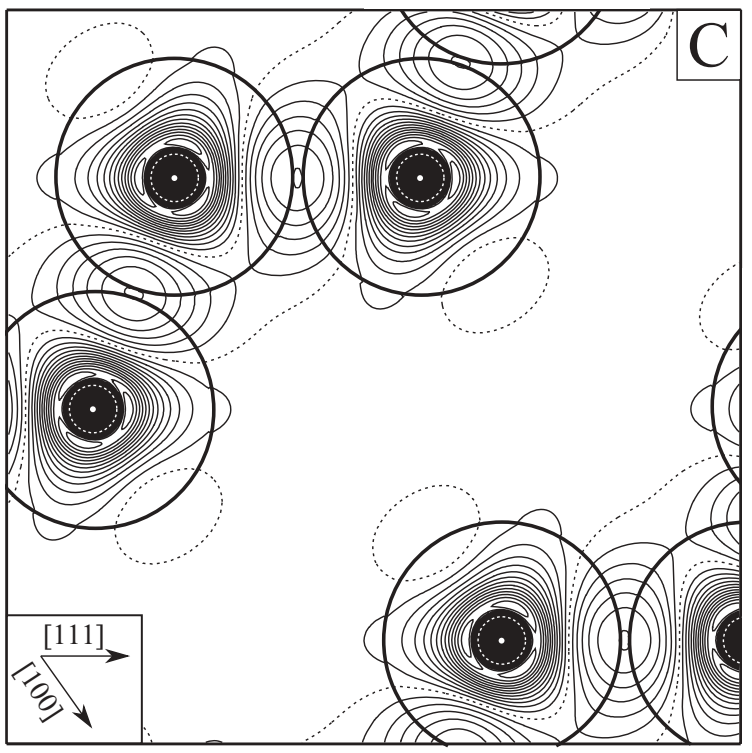

(b)

FIG. 5. (a) Total electron densities obtained from the EXX (solid lines) and LDA potential (dotted lines) on the diamond (01 $\overline{1})$ plane. Contour lines for the values $0.03,0.06, \ldots, 0.99 a_{0}^{-3}$ are shown. (b) Plot of the density difference with contour lines between -0.039 and $0.390 a_{0}^{-3}$ at intervals of $0.003 a_{0}^{-3}$. The dotted line corresponds to $\Delta n(\mathbf{r})=0$.

This becomes clearer in Fig. 5(b), where we plot the difference between the EXX and the LDA densities. The exactly compensated self-interaction allows the charge to accumulate in the atomic cores, but also in the covalent bonds between the atoms.

To understand the requirement of the basis-set balance in more detail, we go back to Eq. (18) whose solution involves the inversion of the response function, Eq. (19). The response function describes the linear response of the electron density with respect to changes of the effective potential. For the latter we employ the MPB, while the former is given by the 


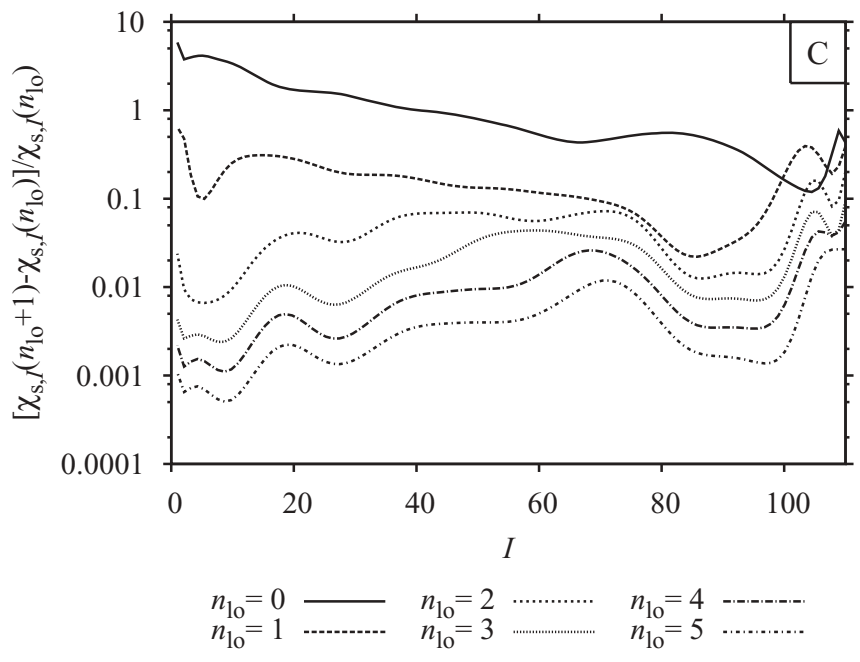

FIG. 6. Convergence of the eigenvalues $\chi_{\mathrm{s}, I}$ of the response function for diamond. The $\chi_{\mathrm{s}, I}$ are all negative and ordered according to increasing moduli. The relative change $\left[\chi_{\mathrm{s}, I}\left(n_{\mathrm{lo}}+1\right)-\right.$ $\left.\chi_{\mathrm{s}, I}\left(n_{\mathrm{lo}}\right)\right] / \chi_{\mathrm{s}, I}\left(n_{\mathrm{lo}}\right)$ is plotted, where $n_{\mathrm{lo}}$ is the number of local orbitals per $l m$ channel $(l=0, \ldots, 5,|m| \leqslant l)$. The case $n_{\mathrm{lo}}=0$ corresponds to the conventional LAPW basis without local orbitals. A Bezier algorithm was employed to smooth the curves.

orbital densities of the occupied states, Eq. (4), and, hence, ultimately by the LAPW basis set. Thus, the LAPW basis must provide enough flexibility for the density to enable it to respond adequately to the changes of the effective potential.

This explains the observed behavior and becomes evident in the convergence of the response function with respect to the LAPW basis. In Fig. 6 we show the changes in the eigenvalues of $\chi_{\mathrm{s}}$, ordered according to increasing moduli, when we add more and more local orbitals, $2 l+1$ additional local orbitals per $l$ quantum number $(l=0, \ldots, 5)$ in each step. We again use $L_{\max }=4$ and $G_{\max }^{\prime}=3.4 a_{0}^{-1}$ as MPB cutoff values. In the case of the maximal number of local orbitals per $l m$ channel, $n_{\mathrm{lo}}=6$, the basis is increased by 432 functions relative to the conventional LAPW basis. Clearly, the eigenvalues can be systematically converged. The relative changes are of the order of $0.1 \%$ to $1.0 \%$ between $n_{\mathrm{lo}}=5$ and $n_{\mathrm{lo}}=6$. The small eigenvalues especially converge well and are particularly important in the inversion of the response function. We note that a straightforward elimination of the small eigenvalues by singular value decomposition is not advisable and leads to an ill-defined response function.

Up to now, we have discussed the importance of the quality of the LAPW basis for the shape of the local EXX potential. Only for well-balanced basis sets is the potential smooth and physical (cf. Fig. 1). We now address the question to what extent this effect influences the KS one-particle energies that result from the self-consistent solution of Eq. (3) with the local EXX potential obtained from the OEP Eq. (13). Table IV gives the transition energies, that is, the KS eigenvalue differences, from the valence-band maximum at the $\Gamma$ point to the $\mathrm{L}$ and $\mathrm{X}$ point of the lowest conduction band of diamond for the basis sets with $n_{\mathrm{lo}}=0, \ldots, 6$ local orbitals per $l m$ channel. Obviously, at least three local orbitals are necessary to converge these transition energies to within $0.01 \mathrm{eV}$. Between
TABLE IV. KS transition energies (in eV) for diamond obtained from the self-consistent solution of the KS equation with the local EXX potential for LAPW basis sets including zero to six local orbitals per $l m$ channel $(l=0, \ldots, 5,|m| \leqslant l)$. As the LAPW basis is made more flexible, the transition energies converge.

\begin{tabular}{lccc}
\hline \hline$n_{\mathrm{lo}}$ & $\Gamma \rightarrow \Gamma$ & $\Gamma \rightarrow \mathrm{L}$ & $\Gamma \rightarrow \mathrm{X}$ \\
\hline 0 & 6.351 & 9.243 & 5.307 \\
1 & 6.196 & 9.086 & 5.125 \\
2 & 6.186 & 9.069 & 5.144 \\
3 & 6.180 & 9.063 & 5.138 \\
4 & 6.178 & 9.059 & 5.139 \\
5 & 6.177 & 9.057 & 5.136 \\
6 & 6.176 & 9.055 & 5.136 \\
\hline \hline
\end{tabular}

the unbalanced and the balanced basis sets $\left(n_{\mathrm{lo}}=0\right.$ and $n_{\mathrm{lo}}=6$, respectively), the values change by about $0.2 \mathrm{eV}$.

Another balance condition we find for the $1 s$ core state, which goes into both the left- and the right-hand sides of Eq. (18). In particular, we now distinguish four cases: (a) core state considered in $\chi_{\mathrm{s}, I J}$ and $t_{I}$, (b) core state considered only in $\chi_{\mathrm{s}, I J}$, (c) core state considered only in $t_{I}$, and (d) core state neglected in both. Case (a) corresponds to the full calculations presented so far. Figure 7 shows that the resulting potentials look very different for the different cases. Surprisingly, potential (d) is closest to the full potential, while potentials (b) and (c) are much too shallow and too strongly varying, respectively. Obviously, the inclusion of the core state on only one side of the OEP equation gives rise to an equation that is out of balance and that yields an unphysical $V_{\mathrm{x}}(\mathbf{r})$. This also influences the resulting KS transition energies. In the balanced case (d), the results $6.19,9.08$, and $5.28 \mathrm{eV}$ for $\Gamma \rightarrow \Gamma, \Gamma \rightarrow \mathrm{L}$, and $\Gamma \rightarrow \mathrm{X}$, respectively, are surprisingly close to the full calculation (a) (cf. Table IV), while the energies for the unbalanced case (b) deviate more strongly, especially for the $\Gamma \rightarrow \mathrm{X}$ transition, $6.22,9.16$, and $6.03 \mathrm{eV}$. The energetic position of the $1 \mathrm{~s}$ state with respect to the Fermi energy, however, is only realistic for the full calculation

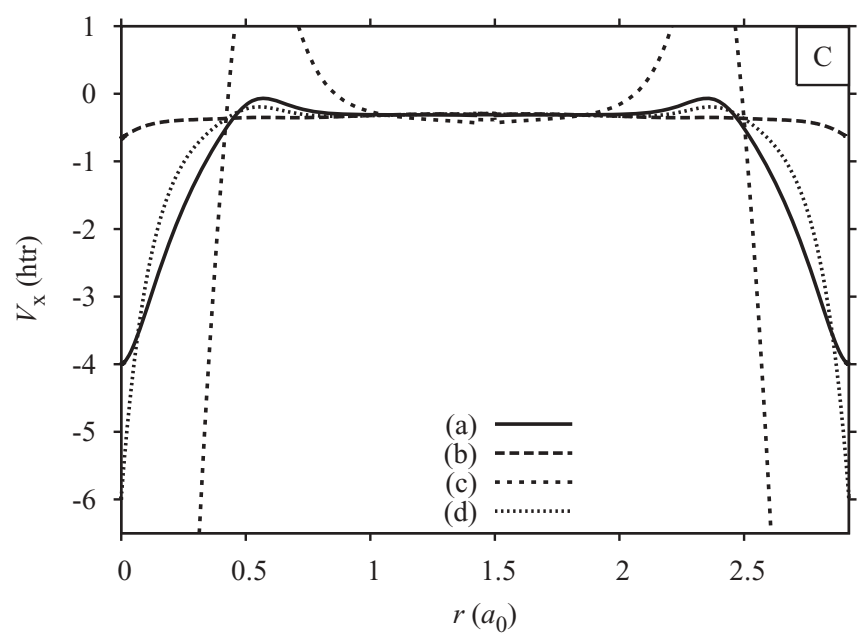

FIG. 7. Same as Fig. 1 for the cases (a) $1 s$ state fully considered, (b) $1 s$ state considered only in $\chi_{\mathrm{s}, I J}$, (c) $1 s$ state considered only in $t_{I}$, and (d) $1 s$ state neglected in both (see text). 
TABLE V. KS transition energies (in eV) obtained with the local EXX and EXX + VWNc potentials and an $8 \times 8 \times 8 \mathbf{k}$-point sampling. For comparison, plane-wave PP results and experimental values from the literature are listed.

\begin{tabular}{|c|c|c|c|c|c|c|c|}
\hline & & \multicolumn{3}{|c|}{ This work } & \multicolumn{2}{|c|}{ Plane-wave PP } & \multirow[b]{2}{*}{ Expt. } \\
\hline & & LDA & EXX & $\mathrm{EXX}+\mathrm{VWNc}$ & EXX & $\mathrm{EXX}+\mathrm{VWNc}$ & \\
\hline \multirow[t]{3}{*}{$\mathrm{C}$} & $\Gamma \rightarrow \Gamma$ & 5.56 & 6.21 & 6.26 & $6.19,{ }^{\mathrm{a}} 6.21^{\mathrm{b}}$ & $6.28^{c}$ & $7.3^{\mathrm{e}}$ \\
\hline & $\Gamma \rightarrow \mathrm{L}$ & 8.43 & 9.09 & 9.16 & $9.15^{\mathrm{b}}$ & $9.18^{\mathrm{c}}$ & \\
\hline & $\Gamma \rightarrow \mathrm{X}$ & 4.71 & 5.20 & 5.33 & $5.34^{\mathrm{b}}$ & $5.43^{c}$ & \\
\hline \multirow[t]{3}{*}{$\mathrm{Si}$} & $\Gamma \rightarrow \Gamma$ & 2.53 & 3.13 & 3.21 & $3.12^{\mathrm{b}}$ & $3.26^{\mathrm{c}}$ & $3.4^{\mathrm{e}}$ \\
\hline & $\Gamma \rightarrow \mathrm{L}$ & 1.42 & 2.21 & 2.28 & $2.21^{\mathrm{b}}$ & $2.35^{\mathrm{c}}$ & $2.4^{\mathrm{e}}$ \\
\hline & $\Gamma \rightarrow \mathrm{X}$ & 0.61 & 1.30 & 1.44 & $1.25^{\mathrm{b}}$ & $1.50^{\mathrm{c}}$ & \\
\hline \multirow[t]{3}{*}{$\mathrm{SiC}$} & $\Gamma \rightarrow \Gamma$ & 6.27 & 7.18 & 7.24 & & $7.37^{\mathrm{c}}$ & \\
\hline & $\Gamma \rightarrow \mathrm{L}$ & 5.38 & 6.14 & 6.21 & & $6.30^{c}$ & \\
\hline & $\Gamma \rightarrow \mathrm{X}$ & 1.32 & 2.29 & 2.44 & & $2.52^{\mathrm{c}}$ & $2.42^{\mathrm{e}}$ \\
\hline \multirow[t]{3}{*}{$\mathrm{Ge}$} & $\Gamma \rightarrow \Gamma$ & -0.14 & 1.24 & 1.21 & & $1.28^{\mathrm{c}}$ & $1.0^{\mathrm{e}}$ \\
\hline & $\Gamma \rightarrow \mathrm{L}$ & 0.06 & 0.89 & 0.94 & & $1.01^{\mathrm{c}}$ & $0.7^{\mathrm{e}}$ \\
\hline & $\Gamma \rightarrow \mathrm{X}$ & 0.66 & 1.15 & 1.28 & & $1.34^{\mathrm{c}}$ & $1.3^{\mathrm{e}}$ \\
\hline \multirow{3}{*}{ GaAs } & $\Gamma \rightarrow \Gamma$ & 0.29 & 1.72 & 1.74 & & $1.82^{\mathrm{c}}$ & $1.63^{\mathrm{e}}$ \\
\hline & $\Gamma \rightarrow \mathrm{L}$ & 0.85 & 1.79 & 1.86 & & $1.93^{\mathrm{c}}$ & \\
\hline & $\Gamma \rightarrow \mathrm{X}$ & 1.35 & 1.95 & 2.12 & & $2.15^{\mathrm{c}}$ & $2.18^{\mathrm{e}}$ \\
\hline \multirow[t]{3}{*}{$\mathrm{Ne}$} & $\Gamma \rightarrow \Gamma$ & 11.43 & 14.79 & 15.46 & $14.15^{\mathrm{d}}$ & $14.76^{\mathrm{d}}$ & $21.51^{\mathrm{f}}$ \\
\hline & $\Gamma \rightarrow \mathrm{L}$ & 16.97 & 20.49 & 21.16 & & & \\
\hline & $\Gamma \rightarrow \mathrm{X}$ & 18.27 & 21.85 & 22.56 & & & \\
\hline \multirow[t]{3}{*}{ Ar } & $\Gamma \rightarrow \Gamma$ & 8.19 & 9.65 & 10.09 & $9.61^{\mathrm{d}}$ & $9.95^{\mathrm{d}}$ & $14.15^{\mathrm{f}}$ \\
\hline & $\Gamma \rightarrow \mathrm{L}$ & 11.06 & 12.22 & 12.60 & & & \\
\hline & $\Gamma \rightarrow \mathrm{X}$ & 10.86 & 12.08 & 12.49 & & & \\
\hline
\end{tabular}

a From Ref. 26.

${ }^{\mathrm{b}}$ From Ref. 48.

${ }^{\mathrm{c}}$ From Ref. 23.

${ }^{\mathrm{d}}$ From Ref. 49.

eFrom Ref. 50.

${ }^{\mathrm{f}}$ From Ref. 51.

(a), $\epsilon(1 s)=-265.7 \mathrm{eV}$ (cf. $-262.9 \mathrm{eV}$ for LDA), whereas calculations (b) and (d) give binding energies that are lower by 31.6 and $10.5 \mathrm{eV}$, respectively. Calculation (c) is unstable and does not converge. These results indicate that the PP approximation is, indeed, suitable for the EXX-OEP approach, a conjecture that is confirmed by our reference calculations later.

As outlined in Sec. II, the construction of a local EXX potential within the KS formalism of DFT is equivalent to the OEP approach of Sharp and Horton, ${ }^{32}$ where the Hartree-Fock total energy is minimized under the constraint that the wave functions feel a local multiplicative potential. This constraint reduces the Hilbert space for the wave functions and, thus, increases the total energy due to the variational principle. In fact, we find that the total energy for diamond obtained with the nonlocal Hartree-Fock potential, Eq. (10), and an $8 \times 8 \times$ 8 k-point set is $0.24 \mathrm{eV}$ per unit cell lower than that of the EXX-OEP approach.

As reference, we report fully converged KS transition energies for a variety of semiconductors and insulators in Table V. All calculations are performed with an $8 \times 8 \times 8$ Brillouin zone sampling and at the experimental lattice constants (C, 6.746 $a_{0}$; Si, $10.26 a_{0}$; SiC, 8.24 $a_{0}$; Ge, $10.67 a_{0}$; GaAs, $10.68 a_{0}$; Ne, $8.44 a_{0}$; Ar, $9.93 a_{0}$ ). Apart from the EXX-only calculations, we also show transition energies obtained with the EXX + VWNc functional in which the LDA correlation functional from Ref. 5 was added to the EXX functional. Table $\mathrm{V}$ shows that both the EXX and the EXX + VWNc functionals yield transition energies much closer to experiment (last column) than the LDA functional (third column). While for semiconductors the resulting energies are even quantitatively in very good agreement with experiment, there are larger discrepancies for the insulators diamond and, in particular, crystalline Ne and Ar. There is only little difference between the EXX and the EXX + VWNc values. The inclusion of the LDA correlation functional does not lead to a definite improvement. The direct band gap $\Gamma \rightarrow \Gamma$ for diamond of $6.21 \mathrm{eV}$ agrees very well with the value $6.18 \mathrm{eV}$ recently reported by Engel, ${ }^{26}$ who used a plane-wave PP approach with cutoff values pushed to the AE limit. It is even identical to the value obtained with a standard valence-only plane-wave PP approach. ${ }^{48}$ In contrast to that, Sharma et al. calculated a much larger value of $6.67 \mathrm{eV}$ with their FLAPW-EXX implementation. For neon, however, there is a somewhat larger discrepancy with the calculation by Magyar et al. ${ }^{49}$ In conclusion, with our EXX-OEP implementation within the AE FLAPW method, we obtain results in very good agreement with previous plane-wave PP calculations, provided that the basis sets for the wave functions and the potential are properly balanced. This shows that the PP approximation is adequate for the EXX-OEP approach at least for the systems examined here, which is at variance with the findings of Ref. 25. 


\section{CONCLUSIONS}

We have developed an all-electron full-potential implementation of the EXX-OEP approach to DFT within the FLAPW method. We analyzed the conditions and requirements on the basis sets and numerical cutoff parameters to obtain reliable and numerically stable results. Based on this knowledge we presented as proof of principle results on KS transition energies for some typical semiconductors, insulators, and noble-gas solids that are in very good agreement with PP results.

The OEP equation is formulated utilizing the mixed product basis (MPB), ${ }^{42-44}$ which has been adjusted for the present purpose: it is augmented with the atomic EXX potential, the constant function is eliminated, and the basis functions are made continuous all over the space. In this basis, the OEP equation becomes an algebraic equation, which is solved for the local EXX potential with standard numerical tools.

For the case of diamond, we have demonstrated that the local EXX potentials are spatially strongly corrugated, which makes a full-potential treatment even more important than in conventional LDA or GGA calculations. Furthermore, the two basis sets, LAPW and MPB, are not independent. They must be properly balanced to obtain a smooth and physical EXX potential over the whole space. In unbalanced cases, the potential shows spurious oscillations, which we have traced back to an insufficiently converged response function, a function that gives the response of the electron density with respect to changes of the effective potential. If the LAPW basis, which parametrizes the electron density, is not flexible enough, the electron density cannot follow the changes of the effective potential that are described by the MPB, leading to a corrupted response function. As a result, the LAPW basis must be converged with respect to a given MPB. Already in the simple case of diamond, we must add six local orbitals at different energies for each $l m$ channel from $l=0$ to $l=5$ to obtain a smooth potential in the spheres. This shows that the LAPW basis must be converged to an accuracy that is far beyond that of conventional LDA or GGA calculations. Similarly, also the LAPW reciprocal cutoff radii must be chosen large enough.

Not surprisingly, the shape of the EXX potentialoscillatory or smooth-has an impact on the resulting $\mathrm{KS}$ transition energies. We find that with properly balanced basis sets, the transition energies for a variety of semiconductors and insulators obtained with the EXX and the EXX + VWNc functionals are in very good agreement with plane-wave PP results from the literature (crystalline neon is an exception). This confirms that the PP approximation works reliably within the EXX-OEP approach. Our finding is in contradiction to a previously published implementation (Ref. 25) based on the FLAPW method, where large discrepancies with PP results were reported.

Currently, reliable AE full-potential EXX-OEP calculations are computationally very demanding, because of the need for large orbital basis sets, which we attribute partly to the fact that the LAPW basis functions depend explicitly on the effective potential. To refine our full-potential implementation of the EXX-OEP approach, we suggest as a task for the future the investigation of schemes that treat the response of the LAPW basis with respect to changes of the potential more efficiently than by employing local orbitals.

\section{ACKNOWLEDGMENTS}

Financial support from the Deutsche Forschungsgemeinschaft through Priority Program 1145 is gratefully acknowledged. *m.betzinger@fz-juelich.de

${ }^{1}$ P. Hohenberg and W. Kohn, Phys. Rev. 136, B864 (1964).

${ }^{2}$ C. Fiolhais, F. Noguiera, and M. A. L. Marques, eds., A Primer in Density Functional Theory, Vol. 620, Lecture Notes in Physics (Springer, Heidelberg, 2003).

${ }^{3}$ W. Kohn and L. J. Sham, Phys. Rev. 140, A1133 (1965).

${ }^{4}$ D. M. Ceperley and B. J. Alder, Phys. Rev. Lett. 45, 566 (1980).

${ }^{5}$ S. H. Vosko, L. Wilk, and M. Nusair, Can. J. Phys. 58, 1200 (1980).

${ }^{6}$ J. P. Perdew, K. Burke, and M. Ernzerhof, Phys. Rev. Lett. 77, 3865 (1996).

${ }^{7}$ J. P. Perdew and Y. Wang, Phys. Rev. B 33, 8800 (1986).

${ }^{8}$ J. P. Perdew and M. Levy, Phys. Rev. Lett. 51, 1884 (1983).

${ }^{9}$ L. J. Sham and M. Schlüter, Phys. Rev. Lett. 51, 1888 (1983).

${ }^{10}$ R. W. Godby, M. Schlüter, and L. J. Sham, Phys. Rev. B 37, 10159 (1988).

${ }^{11}$ M. Grüning, A. Marini, and A. Rubio, J. Chem. Phys. 124, 154108 (2006); Phys. Rev. B 74, 161103 (2006).

${ }^{12}$ S. Kümmel and L. Kronik, Rev. Mod. Phys. 80, 3 (2008), and references therein.

${ }^{13}$ A. Görling, J. Chem. Phys. 123, 062203 (2005).

${ }^{14}$ A. Görling and M. Levy, Phys. Rev. A 50, 196 (1994).
${ }^{15}$ A. Görling and M. Levy, Int. J. Quantum Chem. 56 (Quantum Chem. Symp. 29), 93 (1995).

${ }^{16}$ A. Görling, Phys. Rev. B 53, 7024 (1996); 59(E), 10370 (1999).

${ }^{17}$ J. D. Talman and W. F. Shadwick, Phys. Rev. A 14, 36 (1976).

${ }^{18}$ J. B. Krieger, Y. Li, and G. J. Iafrate, Phys. Rev. A 45, 101 (1992).

${ }^{19}$ Y. Li, J. B. Krieger, and G. J. Iafrate, Phys. Rev. A 47, 165 (1993).

${ }^{20}$ T. Kotani, Phys. Rev. B 50, 14816 (1994).

${ }^{21}$ T. Kotani, Phys. Rev. Lett. 74, 2989 (1995).

${ }^{22}$ M. Städele, J. A. Majewski, P. Vogl, and A. Görling, Phys. Rev. Lett. 79, 2089 (1997).

${ }^{23}$ M. Städele, M. Moukara, J. A. Majewski, P. Vogl, and A. Görling, Phys. Rev. B 59, 10031 (1999).

${ }^{24}$ A. Fleszar, Phys. Rev. B 64, 245204 (2001).

${ }^{25}$ S. Sharma, J. K. Dewhurst, and C. Ambrosch-Draxl, Phys. Rev. Lett. 95, 136402 (2005).

${ }^{26}$ E. Engel, Phys. Rev. B 80, 161205(R) (2009).

${ }^{27}$ A. Makmal, R. Armineto, E. Engel, L. Kronik, and S. Kümmel, Phys. Rev. B 80, 161204(R) (2009).

${ }^{28}$ A. Hesselmann, A. W. Götz, F. D. Sala, and A. Görling, J. Chem Phys. 127, 054102 (2007).

${ }^{29}$ A. Görling, A. Hesselmann, M. Jones, and M. Levy, J. Chem. Phys. 128, 104104 (2008). 
${ }^{30}$ A. Hesselmann and A. Görling, Chem. Phys. Lett. 455, 110 (2008).

${ }^{31}$ [http://www.flapw.de].

${ }^{32}$ R. T. Sharp and G. K. Horton, Phys. Rev. 90, 317 (1953).

${ }^{33}$ V. Sahni, J. Gruenebaum, and J. P. Perdew, Phys. Rev. B 26, 4371 (1982).

${ }^{34}$ E. Wimmer, H. Krakauer, M. Weinert, and A. J. Freeman, Phys. Rev. B 24, 864 (1981).

${ }^{35}$ M. Weinert, E. Wimmer, and A. J. Freeman, Phys. Rev. B 26, 4571 (1982).

${ }^{36}$ H. J. F. Jansen and A. J. Freeman, Phys. Rev. B 30, 561 (1984).

${ }^{37}$ D. Singh, Phys. Rev. B 43, 6388 (1991).

${ }^{38}$ E. E. Krasovskii, A. N. Yaresko, and V. N. Antonov, J. Electron Spectrosc. Relat. Phenom. 68, 157 (1994).

${ }^{39}$ C. Friedrich, A. Schindlmayr, S. Blügel, and T. Kotani, Phys. Rev. B 74, 045104 (2006).

${ }^{40}$ O. K. Andersen, Phys. Rev. B 12, 3060 (1975).

${ }^{41}$ T. Kotani and M. van Schilfgaarde, Solid State Commun. 121, 461 (2002).

${ }^{42}$ M. Betzinger, C. Friedrich, and S. Blügel, Phys. Rev. B 81, 195117 (2010).
${ }^{43}$ C. Friedrich, S. Blügel, and A. Schindlmayr, Phys. Rev. B 81, 125102 (2010).

${ }^{44}$ C. Friedrich, A. Schindlmayr, and S. Blügel, Comput. Phys. Commun. 180, 347 (2009).

${ }^{45}$ E. Engel, S. Keller, A. Facco Bonetti, H. Müller, and R. M. Dreizler, Phys. Rev. A 52, 2750 (1995).

${ }^{46}$ E. Engel, S. Keller, and R. M. Dreizler, Phys. Rev. A 53, 1367 (1996).

${ }^{47}$ E. Engel, A. Facco Bonetti, S. Keller, I. Andrejkovics, and R. M. Dreizler, Phys. Rev. A 58, 964 (1998).

${ }^{48}$ A. Hesselmann and A. Görling, unpublished results. TroullierMartins pseudopotentials constructed from the atomic EXX potential are employed.

${ }^{49}$ R. J. Magyar, A. Fleszar, and E. K. U. Gross, Phys. Rev. B 69, 045111 (2004)

${ }^{50}$ T. C. Chiang and F. J. Himpsel, Band Structure and Core Levels of Tetrahedrally-Bonded Semiconductors, Vol. 23a, LandoltBörnstein-Group III Condensed Matter Numerical Data and Functional Relationships in Science and Technology (Springer-Verlag, Berlin, 1989).

${ }^{51}$ M. Runne and G. Zimmerer, Nucl. Instr. Methods Phys. Res. Sec. B 101, 156 (1995). 